\title{
Editorial
}

\section{Vertical Transmission of SARS-CoV-2: What is the Optimal Definition?}

\author{
Dean A. Blumberg, MD ${ }^{1}$ Mark A. Underwood, MD, MAS ${ }^{1}$ Herman L. Hedriana, MD \\ Satyan Lakshminrusimha, $\mathrm{MD}^{1}$ \\ ${ }^{1}$ Department of Pediatrics, University of California Davis Children's \\ Hospital, Sacramento, California \\ 2 Department of Obstetrics and Gynecology, University of California \\ Davis Medical Center, Sacramento, California
}

Am J Perinatol 2020;37:769-772.

Alzamora et al recently described an interesting report of a neonate born to a mother with severe novel coronavirus 2019 disease (COVID-19) by cesarean section. The infant tested positive for real-time polymerase chain reaction (RT$\mathrm{PCR}$ ) for severe acute respiratory syndrome-coronavirus-2 (SARS-CoV-2) 16 hours after delivery. ${ }^{1}$ During the period of limited 5-day follow-up, this baby did not show an increased antibody titer (immunoglobulin [Ig]-M or IgG). Similar positive cases of suspected neonatal SARS-CoV-2 infection during the first 2 postnatal days have been reported. ${ }^{2}$ While these patients appear to have acquired infection either by intrauterine or intrapartum transmission, establishment of clear definitions for such transmission is warranted (-Fig. 1).

Intrauterine transmission of SARS-CoV-2 has not been convincingly reported to date. ${ }^{3}$ There is limited information available on intrauterine infection earlier in pregnancy with resolution of maternal infection prior to the time of delivery; SARS-CoV-2 is not known to cause chronic infection, therefore neonatal infection is not likely to be active at delivery in this situation, and confirming transmission early in pregnancy will be challenging in the absence of either a phenotype such as congenital Zika or rubella syndrome or a pattern of increased numbers of miscarriages. Recently, miscarriage secondary to SARS-CoV-2 infection at 34 weeks ${ }^{4}$ and at 19 weeks of gestation with positive SARS-CoV-2 through RT-PCR from maternal nasopharynx, placental submembrane, and cotyledon have been reported. ${ }^{5}$

When maternal infection occurs within 14 days before delivery, there is a theoretical risk of intrauterine transmission, since infection may result in viremia potentially leading to infection of the fetus through a disruption in the placental interface or viral particles in the amniotic fluid (-Fig. 1). Although many studies have not detected SARS-CoV-2 in amniotic fluid by RT-PCR, ${ }^{3,6-9}$ a recently published report from Iran described the detection of SARS-CoV-2 in an amni- otic fluid sample obtained during cesarean section from a mother with severe COVID-19 who subsequently died. ${ }^{10}$ The RT-PCR on the nasal and throat swabs in neonate after delivery were negative, but the second test 24 hours later was positive.

Intrapartum or early postnatal infection could occur through exposure of the delivering neonate to infected maternal blood or secretions. Both may be considered as examples of "vertical" transmission. It is important to differentiate mechanisms of potential maternal-fetal transmission, if possible, as timing and route of infection may affect clinical outcomes. In addition, investigational therapies may be found to decrease or eliminate intrauterine transmission. ${ }^{11}$

We start with several underlying assumptions (-Fig. 1) as follows: (1) the incubation period is 1 to 14 days ${ }^{12,13}$; (2) intrauterine infection may potentially occur transplacentally via blood, or via transmission through swallowed or aspirated amniotic fluid; (3) maternal viremia is unlikely during the incubation period $>48$ hours before symptom onset and the likelihood of positive SARS-CoV-2 through RT-PCR in blood samples is low $(<1 \%)$ in COVID-19 patients ${ }^{9}$; $(4)$ intrapartum transmission may potentially occur due to exposure to maternal blood, vaginal secretions, or feces; (5) early postnatal infection may occur via the respiratory route or due to direct contact with the infected mother or other caretakers, or potential transmission through breast milk (however, to date we are not aware of any reports of viral presence in breast milk); and (6) SARS-CoV-2 virus may be transiently detected for up to 24 hours after birth due to superficial contamination or transient viremia (similar to HIV). It is possible that a similar situation may occur following nasal or oral suctioning and/or intubation during neonatal resuscitation in the delivery room leading to introduction or aspiration of maternal secretions into infant's airway. In these cases, the infant's nasopharyngeal swab may be positive for RT-PCR on the first day, but subsequent swabs might be negative and the infant's blood samples
Address for correspondence

Dean A. Blumberg, MD,

Department of Pediatrics,

University of California Davis

Children's Hospital, 2516

Stockton Boulevard,

Sacramento, CA 95817

(e-mail: dablumberg@ucdavis.edu).
Copyright (c) 2020 by Thieme Medical Publishers, Inc., 333 Seventh Avenue, New York, NY 10001, USA. Tel: +1(212) 760-0888.
DOI https://doi.org/ 10.1055/s-0040-1712457. ISSN 0735-1631. 

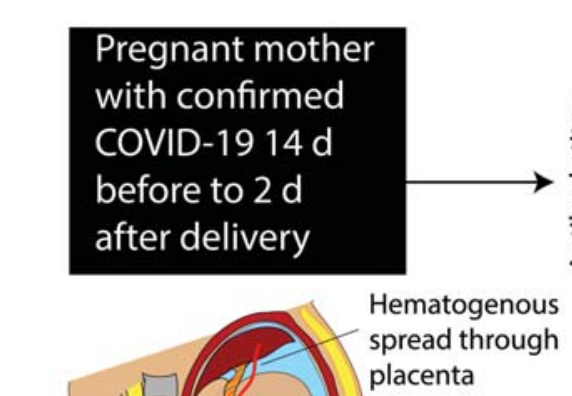

Viral particles in amniotic fluid - inhalation or ingestion by the fetus


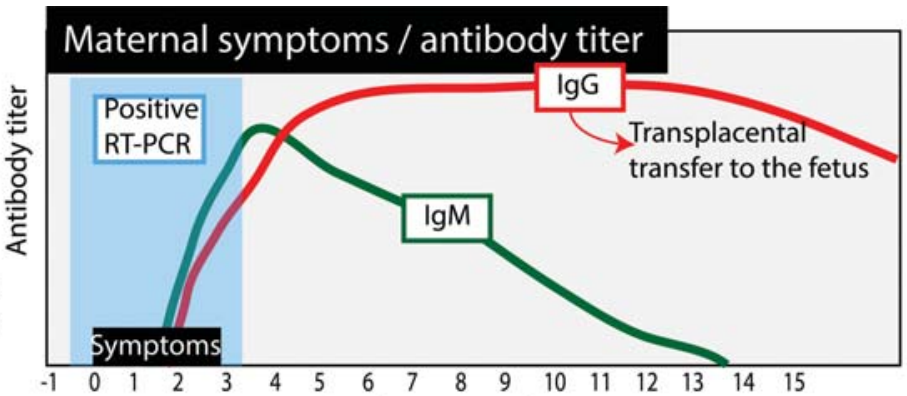

Weeks after Onset of Symptoms

\section{Neonatal Status:}

EARLY EXPOSURE:

Nasopharyngeal RT-PCR positive at $<24 \mathrm{~h}$

RT-PCR positive in amniotic fluid or cord blood or neonatal blood at $<24 \mathrm{~h}$

PERSISTENCE:

Nasopharyngeal RT-PCR positive at $\geq 24-48 \mathrm{~h}$

IgM positive during week 1

Dx: INTRAUTERINE INFECTION

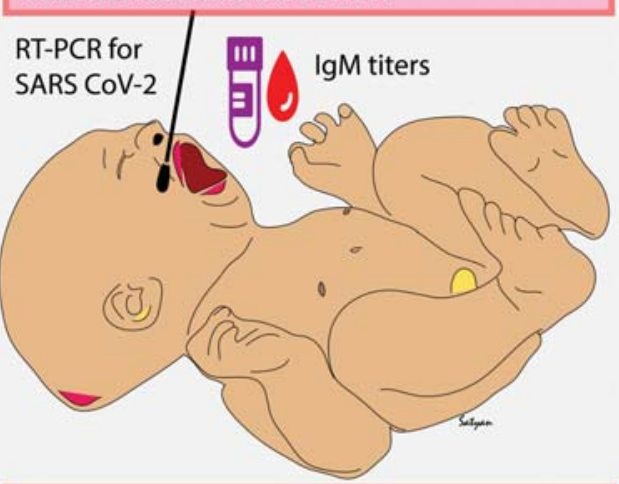

RT-PCR negative at $<24 \mathrm{~h}$ BUT positive 1-14d

after birth

IgM positive at 2-3 weeks

Dx: NEONATAL INFECTION - INTRAPARTUM OR IMMEDIATE POSTNATAL TRANSMISSION

\begin{tabular}{l}
\hline EARLY EXPOSURE: \\
Nasopharyngeal RT-PCR positive at $<24 \mathrm{~h}$ \\
RT-PCR positive in amniotic fluid or \\
cord blood or neonatal blood at $<24 \mathrm{~h}$ \\
NO EVIDENCE OF PERSISTENCE/ IMMUNE \\
RESPONSE: \\
Nasopharyngeal swab negative RT-PCR $\geq 24-48 \mathrm{~h}$ \\
IgM negative during 2 weeks after birth \\
Dx: NEONATAL SUPERFICIAL CONTAMINATION \\
ORTRANSIENT VIREMIA
\end{tabular}

RT-PCR negative at $24-48 \mathrm{~h}$ AND at 7-14d IgM negative during 2 weeks after birth Dx: NO PERINATAL TRANSMISSION

Fig. 1 Peripartum "vertical" transmission of SARS-CoV-2 infection: maternal COVID-19, timeline of infectivity, symptom duration and antibody titers with modes of transmission and neonatal status are shown. The inset graph shows the period of positive testing with nasopharyngeal RTPCR for SARS-CoV-2 in the blue shaded area. The duration of symptoms is shown by the black bar on the horizontal axis. The titers of IgM (green line) and $\mathrm{IgC}$ (red line) in typical patients as described in Li et al ${ }^{16}$ are shown. Potential methods of intrauterine, intrapartum and immediate postnatal transmission are depicted in the left panel. Neonatal testing status with nasopharyngeal RT-PCR and serology titers are shown in the pink box for intrauterine transmission, the orange box for intrapartum or immediate postnatal transmission, the yellow box for superficial contamination/transient viremia and the green box for no evidence of neonatal infection. COVID-19, novel coronavirus disease 2019; Ig, immunoglobulin; RT-PCR, real-time polymerase chain reaction; SARS CoV-2, severe acute respiratory syndrome-coronavirus-2. 
would not show a positive IgM titer (with or without maternal IgG antibodies).

With these assumptions in mind, we propose the following definitions of vertical SARS-CoV-2 transmission ( - Table 1 ).

There are several limitations to the above definition. It is possible for a baby to meet one or more of the early exposure criteria due to contamination with maternal infectious material or transient viremia, and one or more of the persistence criteria due to intrapartum or early postpartum infection with a very short incubation period. In addition, IgM antibodies may be generated as soon as 3 days after symptom onset ${ }^{14,15}$ but may take as long as 2 to 4 weeks. $^{15}$

Table 1 Definitions of vertical SARS-CoV-2 transmission

Intrauterine transmission of SARS-CoV-2 during the peripartum period has likely occurred (even if the neonate is asymptomatic) if there is evidence of both early exposure and persistence, that is, at least one item in each of the following three categories:

1. The mother is positive for SARS-CoV-2 between 14 days prior to birth and 2 days after birth.

2. Early exposure: the virus is detected in any of the following:
(a) A swab of the neonatal respiratory tract (nasopharynx, oropharynx, or saliva) in the first 24 hours of life.
(b) Amniotic fluid.
(c) Umbilical cord blood.
(d) A neonatal blood sample in the first 24 hours of life.

3. Persistence: either of the following:

(a) A swab of the neonatal respiratory tract (nasopharynx, oropharynx, or saliva) is positive after 24 hours of postnatal life.

(b) The neonate has a positive SARS-CoV-2 IgM assay in the first 7 days of postnatal life.

Intrapartum or early postnatal transmission of SARS-CoV-2 has likely occurred (even if the neonate is asymptomatic) if there is lack of evidence of in utero exposure and evidence of intrapartum or early postnatal transmission, that is, at least one item in each of the following three categories:

1. The mother or another person in close contact with the baby is positive for SARS-CoV-2 between 14 days prior to birth and 2 days after birth.

2. Early exposure: a swab of the neonatal respiratory tract (nasopharynx, oropharynx, or saliva) in the first 24 hours of life is negative.

3. Persistence: either of the following:

(a) A swab of the neonatal respiratory tract (nasopharynx, oropharynx, or saliva) is positive between 24 hours and 2 weeks of postnatal life.

(b) The neonate has a positive SARS-CoV-2 IgM assay in the first 2 to 3 weeks of postnatal life.

Superficial exposure to SARS-CoV-2 or transient viremia has likely occurred if the neonate is asymptomatic and at least one item in each of the following three categories is met:

1. The mother is positive for SARS-CoV-2 between 14 days prior to birth and 2 days after birth.

2. Early exposure: the virus is detected in any of the following:
(a) A swab of the neonatal respiratory tract (nasopharynx, oropharynx, or saliva) in the first 24 hours of life.
(b) Amniotic fluid.
(c) Umbilical cord blood.
(d) A neonatal blood sample in the first 24 hours of life.

3. No evidence of persistence or immune response.
(a) A swab of the neonatal respiratory tract (nasopharynx, oropharynx, or saliva) is negative between 24 and 48 hours of life.
(b) The neonate has a negative SARS-CoV-2 IgM assay in the first 2 to 3 weeks of postnatal life.

\section{Recommendation}

As a minimum, a swab of the respiratory tract in the first and second 24-hour periods and a SARS-CoV-2 IgM assay at days 5 to 14 after birth; if initial SARS-CoV-2 IgM is negative, this may be repeated at 2 to 3 weeks of age. 
Therefore, intrauterine transmission occurring late in pregnancy might yield a false-negative result in the first 7 days of life, while intrapartum infection could potentially yield a positive result by day $7 .{ }^{17}$ Finally, our current understanding of IgM dynamics is based on adult data and will be subject to revisiting once neonatal IgM data are available. This definition will likely have decreased sensitivity for prematurely born neonates with more limited capability for IgM production. ${ }^{18}$ Extending the postnatal period for IgM positivity will increase sensitivity at the cost of decreasing specificity for intrauterine infection as detection of postnatal infection is possible after day 7 of life. Finally, the reliability of IgM assays under development is not well known, sensitivities are not clear, nor is the rate of cross-reactivities or false-positive rates. We do not see a role for IgG testing in this situation. Distinguishing between intrapartum and early postnatal transmission of SARS-CoV-2 presents a challenge given the variability in the incubation period and the possibility that asymptomatic family members or health care providers may be shedding the virus at the time of delivery.

This initial definition will likely need refinement but has value for standardizing communications and for clarifying the term "vertical" transmission in distinguishing when possible between intrauterine and intrapartum/postnatal transmission of SARS-CoV-2. Standardized definitions also have implications for future research describing clinical courses and for interrupting transmission.

Conflict of Interest

None declared.

\section{References}

1 Alzamora MC, Paredes T, Caceres D, Webb CM, Valdez LM, La Rosa M. Severe COVID-19 during pregnancy and possible vertical transmission. Am J Perinatol 2020 (e-pub ahead of print). Doi: 10.1055/s-0040-1710050

$2 \mathrm{Hu}$ X, Gao J, Luo X, et al. Severe acute respiratory syndrome coronavirus 2 (SARS-CoV-2) vertical transmission in neonates born to mothers with coronavirus disease 2019 (COVID-19) pneumonia. Obstet Gynecol 2020 (e-pub ahead of print). Doi: 10.1097/AOG.0000000000003926
3 Chen H, Guo J, Wang C, et al. Clinical characteristics and intrauterine vertical transmission potential of COVID-19 infection in nine pregnant women: a retrospective review of medical records. Lancet 2020;395(10226):809-815

4 Liu Y, Chen H, Tang K, Guo Y. Clinical manifestations and outcome of SARS-CoV-2 infection during pregnancy. J Infect 2020;S01634453(20)30109-2

5 Baud D, Greub G, Favre G, et al. Second-trimester miscarriage in a pregnant woman with SARS-CoV-2 infection. JAMA 2020;323 (21):2198-2200

6 Yan J, Guo J, Fan C, et al. Coronavirus disease 2019 (COVID-19) in pregnant women: A report based on 116 cases. Am J Obstet Gynecol 2020;S0002-9378(20)30462-2

7 Yang P, Wang X, Liu P, et al. Clinical characteristics and risk assessment of newborns born to mothers with COVID-19. J Clin Virol 2020;127:104356

8 Liu W, Wang J, Li W, Zhou Z, Liu S, Rong Z. Clinical characteristics of 19 neonates born to mothers with COVID-19. Front Med 2020

9 Wang W, Xu Y, Gao R, et al. Detection of SARS-CoV-2 in different types of clinical specimens. JAMA 2020;323(18):1843-1844

10 Zamaniyan M, Ebadi A, Aghajanpoor Mir S, et al. Preterm delivery in pregnant woman with critical COVID-19 pneumonia and vertical transmission. Prenat Diagn 2020. Doi: 10.1002/pd.5713

11 Dashraath P, Wong JLJ, Lim MXK, et al. Coronavirus disease 2019 (COVID-19) pandemic and pregnancy. Am J Obstet Gynecol 2020 (e-pub ahead of print). Doi: 10.1016/j.ajog.2020.03.021

12 Lauer SA, Grantz KH, Bi Q et al. The incubation period of coronavirus disease 2019 (COVID-19) from publicly reported confirmed cases: estimation and application. Ann Intern Med 2020;172(09):577-582

13 Rasmussen SA, Smulian JC, Lednicky JA, Wen TS, Jamieson DJ. Coronavirus Disease 2019 (COVID-19) and pregnancy: what obstetricians need to know. Am J Obstet Gynecol 2020;222(05): 415-426

14 Dong L, Tian J, He S, et al. Possible vertical transmission of SARSCoV-2 from an infected mother to her newborn. JAMA 2020

15 Zeng H, Xu C, Fan J, et al. Antibodies in infants born to mothers with COVID-19 pneumonia. JAMA 2020;323(18):1848-1849

16 Li G, Chen X, Xu A. Profile of specific antibodies to the SARSassociated coronavirus. N Engl J Med 2003;349(05):508-509

17 Kimberlin DW, Stagno S. Can SARS-CoV-2 infection be acquired in utero?: More definitive evidence is needed JAMA 2020 (e-pub ahead of print). Doi: 10.1001/jama.2020.4868

18 Ballow M, Cates KL, Rowe JC, Goetz C, Desbonnet C. Development of the immune system in very low birth weight (less than $1500 \mathrm{~g}$ ) premature infants: concentrations of plasma immunoglobulins and patterns of infections. Pediatr Res 1986;20(09):899-904 